\title{
Modeling and Stochastic Control for Home Energy Management
}

\author{
Zhe $\mathrm{Yu}^{\dagger}$, Linda McLaughlin ${ }^{\dagger}$, Liyan Jia ${ }^{\dagger}$, Mary C. Murphy-Hoye ${ }^{\ddagger}$, Annabelle Pratt ${ }^{\ddagger}$, and Lang Tong ${ }^{\dagger}$
}

\begin{abstract}
The problem of modeling and control for Home Energy Management (HEM) is considered. A first order thermal dynamic model is considered and its parameters are extracted using real measurements over a period of three summer months. The identified model is validated using separate data sets. The extracted model shows certain nonstationarity and non-Gaussianity. However, local approximations using a stationary model are shown to have relatively small modeling and prediction errors. The extracted model is then used for developing a multi-scale multi-stage stochastic optimization framework for the control of the Heating, Ventilation, and Air Conditioning (HVAC) unit, the charging of Plug-in Hybrid Electric Vehicle (PHEV), and the scheduling of deferrable load such as washer/dryer operations. A two time scale Model Predictive Control (MPC) strategy is proposed that minimizes the discomfort level subject to power and budget constraints: at the slow time scale, a power budget is allocated across different appliances at the hourly level; at the fast time scale, sensor measurements are used for the scheduling and control of different loads. Using parameters extracted from the real data, the proposed approach is compared with the simple rule based control strategy typically used in HVAC controllers.

Index Terms-Home energy management, model predictive control, smart grid, demand response, HVAC control, stochastic optimization.
\end{abstract}

\section{INTRODUCTION}

W E consider the problem of managing residential energy consumption as part of the future smart grid through a Home Energy Manage (HEM) device. While there is not yet a well defined smart grid architecture at the distribution network level, there seems to be a consensus that demand side response will be an essential feature. In particular, an effective demand response should include the ability to shift loads when possible, to react to dynamic price changes, and save money while maintaining the preferred comfort level of the residence.

A HEM device plays the role of an in-house control center that collects sensor measurements, schedules various appliances and loads, and interacts with the energy infrastructure outside the residential home. We consider in this paper a particular form of interaction in which the HEM device is given the power limit, the hourly maximum power consumption for example, by which the HEM device must schedule the in house appliances so that the total power consumption is below the prescribed limit. The main advantage of using such power limit as the interface is that it allows the HEM device to manage the

${ }^{\dagger}$ Z. Yu, L. Mclaughlin, L. Jia, and L. Tong are with the School of Electrical and Computer Engineering, Cornell University, Ithaca, NY 14853, USA. Email: $\left\{\right.$ zy73, lnm36, 1j92, 1t35\}@cornell.edu. ${ }^{\ddagger}$ A. Pratt and M. Murphy-Hoye and are with the Intel Corp., USA.

This work is supported in part by the National Science Foundation under Grant CNS1135844 and the Intel Fellowship Program. consumption according to the customer's preference as oppose allowing the utility directly controls the air conditioning or the refrigerator unit.

The power limit that serves as the key constraint in HEM can be signaled directly by the utility either at a time of power shortage or as part of a long term energy saving plan. Alternatively, the power limit can also be the result of market design. In the context of a hierarchical demand side management (DSM) system, for example, an energy aggregator interfaces with a Retail Electric Provider (REP) and a pool of customers. In a hypothetical DSM operation, as illustrated in Fig. 1, the aggregator secures a contract with the REP and promises an aggregated load profile among its customers. The aggregator incentivizes its customers by a certain pricing scheme to fulfill the promised load profile. The result is that each customer provides the aggregator with its own load profile that specifies the maximum power consumption. In the event of required load shifting, the aggregator may send requests of changes in individual load profiles, and the HEM device of the customer can respond accordingly.

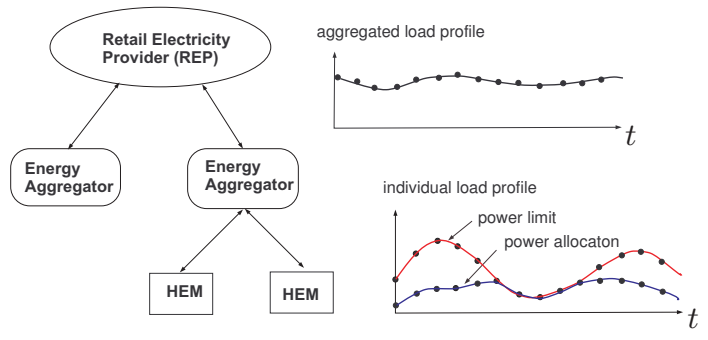

Fig. 1: A hierarchical demand side management system

An essential function of an HEM device is to manage energy consumption based on the load profile promised to the aggregator, the pricing signal from the aggregator, the sensing devices that measure the home environment, the desired comfort level, and budget constraints. The customer may specify a certain monthly energy expenditure and expect the HEM device to optimize intelligently energy usage that fits the customer's specific lifestyle.

\section{A. Summary of results}

This paper contains two parts. First, we present results of the modeling of the thermal dynamics of a residential house based on real data collected during a three month period in Arizona. We use a physics-based model, leading to a first 
order approximation. Our investigation reported in Section III includes both model identification and validation.

The use of simple first order linear dynamic model allows us to develop stochastic control algorithms, capable of scheduling not only the HVAC but also other types of loads. Our data analysis shows that, although the thermal dynamic model appears to be nonstationary, the linear time invariant (LTI) model approximates reasonably well within the time window of 24 hours. The real data also shows the weaknesses of the LTI model. In particular, the modeling residue error is shown to be non-Gaussian and non-white; its power spectrum contains periodic components.

The second part is a computationally tractable multi-scale multi-stage optimization framework that integrates various functionalities of HEM. The multi-scale multi-stage nature of the problem arises naturally in home energy management. For example, the thermal dynamics of a residential home may be modeled at the minute level, the load profile for the maximum power consumption is specified at the hourly level, and the targeted energy expenditure is given at weekly or monthly level. The scheduling of various devices also involves multiple stages as information required for scheduling arrives at different time, which affects the time when scheduling decisions are made. Unfortunately, finding the optimal scheduling policy of a multi-scale multi-stage stochastic program is intractable in general.

We propose a suboptimal but computationally tractable approach based on the principle of Model Predictive Control (MPC) [1]. The HEM device collects sensor data based on which it predicts the energy state of the physical plant into the future and makes tentative future decisions optimally based on such forecasts. The key of MPC is to exercise only the immediate tentative decision and refine its forecast as it collects more information. MPC does not lead to the optimal solution in general, but the strategy is widely used in practice; it is computationally tractable and often offers satisfactory performance.

We propose a hierarchical structure that separates the problem into slow and fast scale optimizations; the former provides the hourly power (energy) budget for different devices whereas the latter determines control signals at the time scale that matches to that of the thermal dynamics (minute level). The proposed approach also separates continuous variable from integer variable optimizations. To provide power allocation at the slow time scale, the optimization involves a quadratic optimization with linear constraints, which can be solved easily using standard numerical techniques. At the fast time scale, because of the on-off nature of HVAC control, the problem becomes a stochastic integer program where a suboptimal heuristic approach is proposed.

Real data is used in evaluating the performance of the proposed approach based on both stationary and non-stationary models. Numerical results show gains at different levels over the standard rule-based HVAC control strategy.

\section{B. Related work}

The literature on home energy management is extensive and expanding. We focus here related work on the modeling and control aspects of HEM. The literature on thermal dynamics models dates from 1978. The work reported in [2] used a convenient set of equivalent thermal parameters for residential townhouse. Our approach is mostly closely related to [3] where a dynamic model of the response of a single residential air conditioner load to weather conditions is developed. This approach uses estimation techniques and an air conditioner load model based upon equations for energy balance and mass balance for the air inside a customer's residence. In [4], an identification algorithm for load models have been proposed exploiting the alternating renewal nature of the thermostat switching process. The author in [5] propose a method for estimation of continuos-time models using the maximum likelihood method and a kalman filter to calculate the likelihood function.

There is also substantial literature on temperature control, but few published work provides an integrated approach to loads of different types, addressing design tradeoffs among comfort requirements and peak power and budget constraints. Authors of [6] proposed a three-layer control mechanism and use Tabu search to find a feasible solution. In [7], particle swarm optimization is used to find the optimal solution for coordinately scheduling multiple energy resources. These approaches require accurate prediction of the energy usage of future. In [8], uncertainty consideration is incorporated into the optimization, but the control is an open loop strategy without using the real-time measurement.The authors of [9] considered a similar scheduling problem as one treated in this paper. The emphasis in [9] is on the tradeoff between cost and waiting time in a multi-home setting. The work presented here, in contrast, focuses on energy management problem within a single home with a design tradeoff between cost and comfort level subject to budget and power constraints. It is also significant that the scheduling problem considered in this paper involves thermal dynamics that dictates the formulation of multi-stage stochastic dynamic optimization. The thermal dynamics is not modeled in [9] and the optimization involved is considerably simpler. The thermal dynamics are included in the HEM proposed for a single home in [10], using a direct search optimization. It is a single stage optimization which sets the thermostat set points, whereas this work includes direct control of the HVAC system.

The MPC strategy adopted in this paper goes back to [11], [12], [13]. In [11], an algorithm referred to as LQGMPC was proposed to deal with the state and control linear inequality constraints. In [12], [13], the Quadratic Dynamic Matrix Control is used to solve nonlinear process optimization with state estimation.

In [14] we proposed a preliminary algorithm based on the MPC method. Simulations were carried out using virtual parameters. In this paper, modeling part is included and comparison with real HVAC performance is presented. 


\section{Organization and Notations}

This paper is organized as follows. Section II introduces a stochastic optimization framework. Section III presents the thermal dynamic model identification and validation based on real measurements. In Section IV, we propose a multi-stage multi-scale approach to HEM followed by numerical results in Section V.

Notations used in this paper are standard. Because multiple time scales are involved, we adopt the notation that $x[t]$ denotes the fast scale signal (say in minutes scale) and $x_{t}$ stands for the signal at the slow scale (hourly variables).

\section{A STOCHASTIC OPTIMIZATION FRAMEWORK FOR HEM}

In this section we present the basic residential model, including different load classes, the thermal dynamic model, and performance measure. We then formulate the HEM scheduling problem as one of stochastic program.

\section{A. Load classes and characteristics}

Three types of loads are considered in this paper: The first is the HVAC units, which are scheduled by the HEM device to control the indoor temperature $x^{\text {in }}[t]$ for comfort. The HEM device collects sensor measurements, obtains model parameters, and schedules appliances using the algorithms presented in Section IV.

The second load type is the deferrable and interruptible load. The specific example considered in this paper is the charging of PHEV for which the earliest starting and the latest completion times are specified; the actual charging time and the amount of charging are part of the optimization. We assume that the charging can be suspended temporarily and resumed at a later time. The strategy of charging affects the customer comfort level indirectly through the peak power constraints and available budget. PHEV is required to be charged to a certain level by the deadline, which stands for a higher priority over the comfort level. This is similar to the approach used in [10], which takes into account only the cost of charging the battery and not the equivalent cost of battery degradation. A method to include battery degradation is presented in [15]

The third type of load is deferrable and non-interruptible load. The scheduling of a dryer is considered as an example in this paper. Based on the power consumption and the price information, the starting time of the load can be moved within the earliest start time $T_{E}$ and the latest start time $T_{L}$. We assume the HEM knows the load characteristics (power drawn from the grid over time). As with the control of charging, the schedule of the deferrable load affects the comfort level indirectly and it is a hard requirement.

\section{B. Thermal dynamic model}

An electric heating-cooling thermal dynamic based upon the energy balance analysis is used, as discussed in [4] and [16]. The first-order stochastic differential equation of the continuous temperature state $x_{t}$ is stated as following:

$$
d x_{t}^{\text {in }}=a\left(x_{t}^{\text {out }}-x_{t}^{\text {in }}\right) d t+R p_{t}^{\mathrm{HVAC}} d t+\sigma d v_{t}
$$

Where $a$ is the average thermal resistance per thermal capacity of the dwelling in watts/joules, $R$ is the power rating per thermal capacity of the dwelling in watts, and $\sigma v_{t}^{\prime}$ is a wiener process with intensity $\sigma$. The wiener process accounts for heat gain or heat loss such as fluctuating number of people in the residence, doors and windows being opened and closed, refrigerators and cooking, etc

The discrete-time equivalent equation for the continuous physical model in equation (1) is given by

$$
x^{\text {in }}[t+1]=x^{\text {in }}[t]+\alpha\left(x[t]^{\text {out }}-x^{\text {in }}[t]\right)+G p^{\mathrm{HVAC}}[t]+c+w[t]
$$

where

$x^{\mathrm{in}}[t+1]$ is the temperature at time $(t+1)$

$x^{\mathrm{in}}[t]$ is the indoor temperature at time $t$

$x[t]^{\text {out }}$ is the outdoor temperature at time $t$

$\alpha, G, c$ are the essential parameters to estimate

$p^{\mathrm{HVAC}}[t]$ is power consumed by the HVAC

$w[t]$ is the noise modeling

In a state space form with the possibility including multiple HAVC units and multiple sensors, we have

$$
\begin{aligned}
\Phi(A, G, C): & x[t+1]=A x[t]+G p^{\mathrm{HVAC}}[t]+v[t] \\
& y[t]=C x[t]+w[t] .
\end{aligned}
$$

where the state vector $x[t+1] \triangleq\left(x^{\text {in }}[t], x^{\text {out }}[t]\right)$ consists of the indoor temperature $x^{\text {in }}[t]$ and outdoor temperature $x^{\text {out }}[t]$. The multiple HVAC are controlled via vector $p^{\mathrm{HVAC}}[t] . y[t]$ is the measurement and the model includes the process noise $v[t]$ as well as measurement noise $w[t]$.

\section{Performance measure and constraints}

A control/scheduling policy $\pi$ is a power allocation to the three types of loads $\left(p_{\mathrm{HVAC}}^{\pi}[t], p_{\mathrm{PHEV}}^{\pi}[t], p_{\mathrm{Def}}^{\pi}[t]\right)$ at the fast time scale (minute intervals) using measurement $\mathscr{Y}_{[0: t)}$ up to $t$.

Let $\mathscr{P}$ be the set of admissible policies that satisfy the scheduling constraints including the required start-end time for charging and start time for deferrable loads. Of particular importance is the the vector of aggregated hourly load constraints $\left(P_{1}, \cdots, P_{T_{h}}\right)$. Specifically, given a control policy $\pi \in \mathscr{P}$, let $p^{\pi}[t] \triangleq p_{\mathrm{HVAC}}^{\pi}[t]+p_{\mathrm{PHEV}}^{\pi}[t]+p_{\text {Def }}^{\pi}[t]$ be the aggregated power consumption in interval $t$. The hourly power consumption limits mandates that

$$
p_{i}^{\pi}=\sum_{t \text { in hour } i} p^{\pi}[t] \leq P_{i}, \quad i=0,1, \cdots, T_{h} .
$$

Given the price signal $z[t]$ and the time interval $T$, the cost of a policy $\pi$ is give by

$$
\mathcal{C}(\pi) \triangleq \sum_{t} z[t] \times T p^{\pi}[t] .
$$

We measure the (lack of) quality of a policy $\pi \in \mathscr{P}$ by the discomfort level by

$$
\mathcal{D}(\pi) \triangleq \mathbb{E}_{\pi}\left(\sum_{t}\left\|x^{\mathrm{in}}[t]-d[t]\right\|^{2}\right),
$$


where $x^{\text {in }}[t]$ is the indoor temperature and $d[t]$ the desired temperature settings. Given a daily budget constraint $B$, the optimal policy is the solution of the following constrained optimization

$$
\min _{\pi \in \mathscr{P}} \mathcal{D}(\pi) \quad \text { subject to } \quad \mathcal{C}(\pi) \leq B
$$

\section{Model IdENTIFICATION AND VALIDATION}

\section{A. Data collection and measurements}

The data used for this work includes indoor and outdoor temperatures as well as HVAC power usage measurements. The data was collected every 15 minutes during August to November 2010. Fig. 2 shows the floor plan of the house where the data was obtained. The house has 2 floors and uses 3 HVACs as shown in Fig. 2. The measurements were collected from 7 different rooms including the living room, dining room, kitchen, family room, extra room, and 1st floor bedroom. On the second floor, we have data available for the bedroom located on the left side and master bathroom.

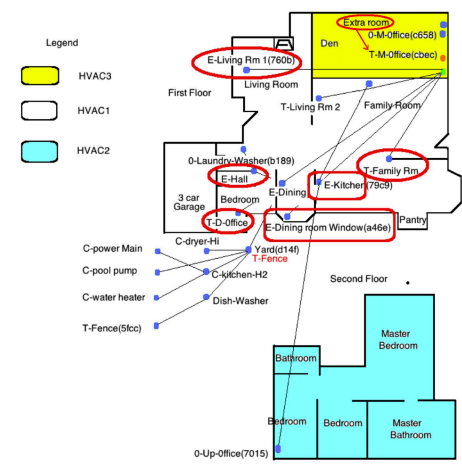

Fig. 2: Floor plan of a residential home

\section{B. Model identification and validation}

The parameters in model equation (2) can be obtained using the method of least squares:

$$
\begin{aligned}
(\hat{c}, \hat{\alpha}, \hat{G})= & \underset{c, \alpha, G}{\operatorname{argmin}}\left(\sum_{t=1}^{N} \|\left(x^{\mathrm{in}}[t+1]-x^{\mathrm{in}}[t]\right)\right. \\
& \left.-\left(c+\alpha\left(x^{\text {out }}[t]-x^{\mathrm{in}}[t]\right)+G p[t]\right) \|^{2}\right),
\end{aligned}
$$

where $\mathrm{N}$ is the number of observations used in the parameter estimation. For time varying models, $N$ should be large enough to obtain reliable estimates but small enough for the model remains stationary. In our study, we considered the cases where $N$ corresponds to 1,7 , and 14 days of measurements.

The accuracy of the model can be measured by the mean squared Modeling Error (ME)

$\varepsilon_{m}=\frac{1}{N} \sum_{t=1}^{N}\left\|\left(x^{\text {in }}[t+1]-x^{\text {in }}[t]\right)-\left(\hat{c}+\hat{\alpha}\left(x^{\text {out }}[t]-x^{\text {in }}[t]\right)+\hat{G} p[t]\right)\right\|^{2}$

The model extracted from the data needs to be validated using different data sets. With the estimated parameters $\hat{\alpha}, \hat{G}, \hat{c}$ obtained from one data set, we test the accuracy of the model using a different data set based on the one step prediction

$$
\hat{x}^{\text {in }}[t+1]=x^{\text {in }}[t]+\hat{\alpha}\left(x^{\text {out }}[t]-x^{\text {in }}[t]\right)+\hat{G} p[t]+\hat{c},
$$

The mean squared Prediction Error (PE) is given by

$$
\varepsilon_{p}=\frac{1}{N} \sum_{t=1}^{N}\left\|\hat{x}^{\mathrm{in}}[t+1]-x^{\mathrm{in}}[t+1]\right\|^{2} .
$$

Table I shows the mean squared modeling error (ME) and mean squared prediction error (PE) for the case when 24 hour of data are used in parameter estimation. More extensive results can be found in [17]. It was observed that both ME and PE are reasonably small with less than 0.1 degree of standard deviation, comparing with the average temperature of around 80 degrees. One particular anomaly is the model fitting for the extra room, which showed very large prediction error. One of the reasons for this anomaly may be that this room was controlled manually, a factor we did not consider in our model.

TABLE I: Mean Squared Modeling and Prediction errors

\begin{tabular}{ccccc}
\hline \hline Room & $\bar{x}_{t}^{\text {In }}\left({ }^{\circ} \mathrm{F}\right)$ & $\sigma_{x_{t}^{\text {in }}}$ & $\varepsilon_{m}$ & $\varepsilon_{p}$ \\
\hline Living Room & 79.1244 & 1.8073 & 0.0103 & 0.0116 \\
Family Room & 80.5193 & 2.5919 & 0.0249 & 0.0282 \\
Kitchen & 81.8173 & 2.1409 & 0.0247 & 0.0281 \\
Dining Room & 79.0615 & 14.0744 & 0.1355 & 0.1480 \\
Office Room & 79.8312 & 9.6645 & 0.0879 & 0.0956 \\
Hall & 79.2099 & 1.1447 & 0.0182 & 0.0212 \\
Extra Room & 83.0398 & 48.2190 & 0.4122 & 29.8200 \\
Ups. Office & 78.2068 & 1.1281 & 0.0184 & 0.0199 \\
Ups. bath & 77.8422 & 2.6523 & 0.0372 & 0.0412 \\
\hline
\end{tabular}

Table II shows the thermal parameters for every room. The standard deviation is relatively large comparing to the mean value, indicating that the confidence interval is not tight.

TABLE II: Thermal Parameters

\begin{tabular}{cccc}
\hline \hline Room & $\bar{\alpha} \pm \sigma_{\alpha}$ & $G \pm \sigma_{G}\left({ }^{\circ} \mathrm{F} / \mathrm{kWh}\right)$ & $\bar{c} \pm \sigma_{c}\left({ }^{\circ} \mathrm{F}\right)$ \\
\hline Living Room & $0.0083 \pm 0.0046$ & $-0.2076 \pm 0.1211$ & $0.0216 \pm 0.0552$ \\
Family Room & $0.0127 \pm 0.0046$ & $-0.1798 \pm 0.1608$ & $0.0062 \pm 0.0908$ \\
Kitchen & $0.0121 \pm 0.0048$ & $-0.1997 \pm 0.1901$ & $0.0324 \pm 0.0894$ \\
Dining Room & $0.0209 \pm 0.0127$ & $-0.5438 \pm 0.1768$ & $-0.0047 \pm 0.1201$ \\
Office Room & $0.0168 \pm 0.0120$ & $-0.5095 \pm 0.2337$ & $0.0176 \pm 0.0802$ \\
Hall & $0.0101 \pm 0.0051$ & $-0.3314 \pm 0.1787$ & $0.0264 \pm 0.0669$ \\
Extra Room & $0.0474 \pm 0.0388$ & $-5.8284 \pm 24.6484$ & $0.0709 \pm 0.3141$ \\
Ups. Office & $0.0107 \pm 0.0048$ & $-0.2990 \pm 0.1214$ & $0.0631 \pm 0.0521$ \\
Ups. bath & $0.0152 \pm 0.0070$ & $-0.4435 \pm 0.6012$ & $0.0417 \pm 0.0820$ \\
\hline
\end{tabular}

Fig. 3 below shows plots for parameter, prediction error, and temperatures evolution, respectively. The estimated parameters were shown to be time varying, indicating model nonstationarity. Fig. 4 shows the predicted and actual temperature based on the proposed LTI approximation using parameters extracted 
from data in the previous 24 hours. It is apparent that the LTI model gave a reasonably accurate approximation.

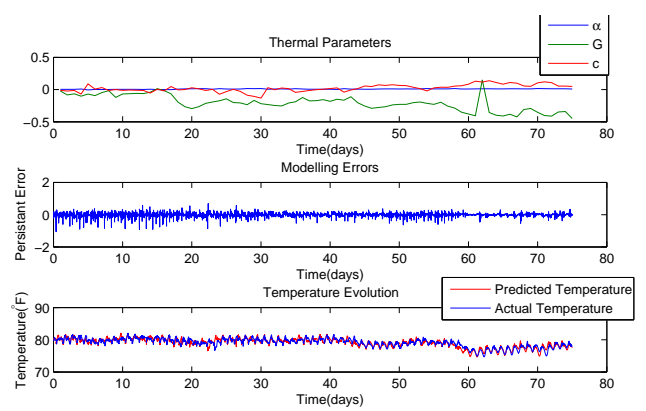

Fig. 3: Parameter expansion over time, prediction error and temperature evolution for living room

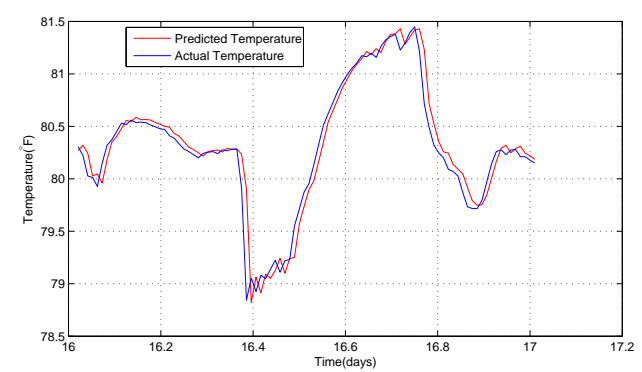

Fig. 4: Temperature prediction using estimated thermal parameters

One way to validate the model is to examine the residue error sequence. The power spectral density of the modeling and prediction error are shown in Fig. 5. The spikes in the estimated power spectral density indicated the presence of harmonics.
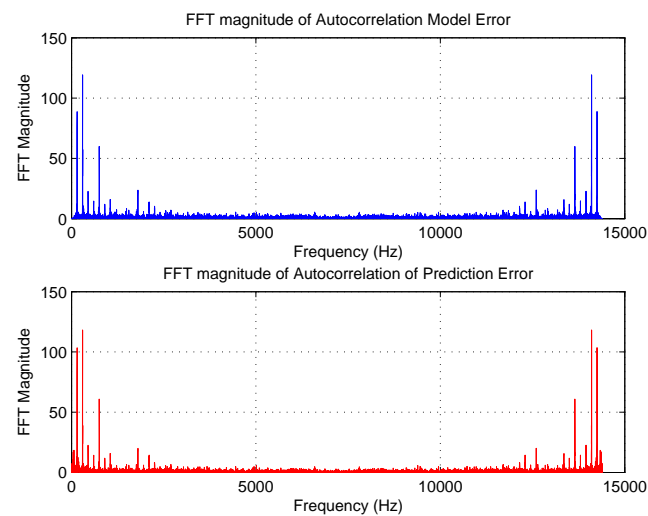

Fig. 5: FFT of Autocorrelation of ME and PE

Another important factor in model validation is the Gaussianity of the residue error. Fig. 6 shows the cumulative distribution functions (CDF) for both modeling and prediction errors, respectively, plotted against the Gaussian CDF. While
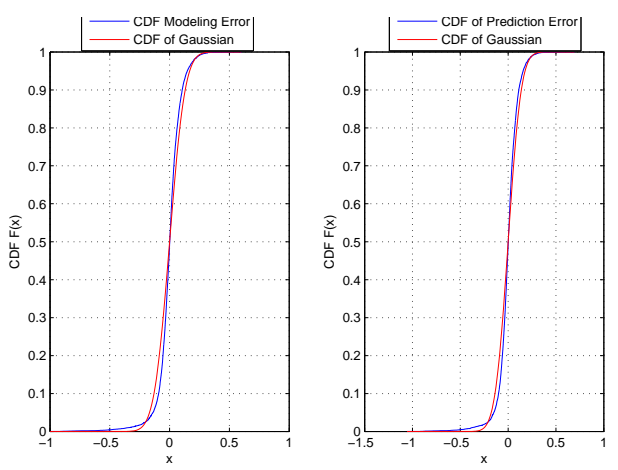

Fig. 6: CDF of ME and PE

the CDF of the residue sequence appeared to be close to Gaussian, a careful examination using the Q-Q plots and Kolgomorov-smirnov test indicated that the residue error is non-Gaussian. See [17] for details.

\section{A MODEL PREDICTIVE CONTROL APPROACH TO HEM}

\section{A. Stochastic programming formulation}

With thermal parameters abstracted from the measurement, we formulate the HEM control as a multi-stage stochastic optimization problem [18]. The goal is to minimize the user's discomfort level subject to budget constraints and peak power limit:

$$
\begin{aligned}
& \operatorname{minimize} \quad \sum_{t=1}^{T_{h}+1} \mathbb{E}_{\pi}\left\|x_{t}^{\text {in }}-d_{t}\right\|^{2} \\
& \text { subject to }\left(x_{t}, y_{t}\right) \sim \Phi(A, G, C) \\
& 0 \leq p_{t}^{\mathrm{HVAC}} \leq P_{\max }^{\mathrm{HVAC}} \\
& T_{E} \leq s \leq T_{L} \\
& p_{t}^{\text {Def }}= \begin{cases}P_{\text {rate }}^{\text {Def }} & \text { if } s \leq t<s+d \\
0 & \text { o.w. }\end{cases} \\
& 0 \leq p_{t}^{\mathrm{PHEV}} \leq P_{\max }^{\mathrm{PHEV}} \\
& \sum_{t=0}^{t=T_{h}-1} T p_{t}^{\mathrm{PHEV}}=Q \\
& p_{t}^{\mathrm{PHEV}}=0 \text { if } t<T_{A} \text { or } t>T_{D} \\
& 0 \leq p_{t}^{\mathrm{HVAC}}+p_{t}^{\mathrm{PHEV}}+p_{t}^{\mathrm{Def}} \leq P_{t} \\
& \sum_{t=0}^{T_{h}-1} z_{t} T\left(p_{t}^{\mathrm{HVAC}}+p_{t}^{\mathrm{PHEV}}+p_{t}^{\mathrm{Def}}\right) \leq B
\end{aligned}
$$

where the indoor temperature vector $x_{t}^{\text {in }}$ is part of the state evolution $\left(x_{t}, y_{t}\right) \sim \Phi(A, G, C)$ specified by the stochastic thermal dynamic equation (3).

A control policy $\pi$ maps the measurements $\mathscr{Y}_{0, n}=$ $\left\{y_{n}, y_{n-1}, \cdots, y_{0}\right\}$ to decision variables $\left(p_{n}^{\mathrm{HVAC}}, p_{n}^{\mathrm{PHEV}}, s\right)$, where $s$ is the start time of the deferrable and non-interruptible load. Note that once the start time is decided, the detailed power allocation is determined.

The stochastic comes from the modeling error as well as the measurement noise, which make the optimization not computationally tractable.

\section{B. Stochastic optimization for HEM}

We propose a multi-scale control architecture of HEM shown in Fig. 7 based on the principle of model predictive 
control (MPC). The detailed optimization functions of slow and fast time scale are stated in Sections IV-D and IV-E.

The measurements (indoor and outdoor temperatures) $y[t]$ is taken at fast time scale. Thermal parameters are obtained by the modeling fitting using the past measurements. With estimated parameters and temperature states data the Kalman filter predict the thermal dynamic states $\hat{x}[t+k \mid t]$ into the future. Both states prediction and the pricing signal $z[t]$ are used in slow time scale MPC to distribute the power budget to different loads. The detailed power allocation is controlled in the fast time scale problem.

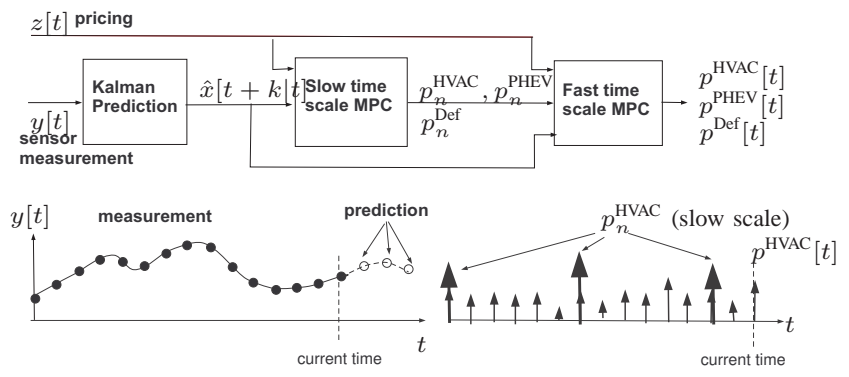

Fig. 7: Multi-scale HEM architecture

\section{Weekly Budget Allocation}

Given the weekly or monthly total budget, the HEM will distribute the money to everyday according to the predicted ambient temperature. Two different methods are considered in this paper. One direct way is to allocate the budget in proportion to the daily average outdoor temperature. The other is known as water filling in wireless communication. The problem is to allocate power to multi channels.Here, we allocate the budget similarly. The money is distributed to every particular day by solving the following optimization problem.

$$
\begin{array}{ll}
\operatorname{maximize} & \sum_{d=t}^{T} \log \left(1+\frac{B_{d}}{T_{d}}\right) \\
\text { subject to } & \sum_{d=t}^{T} B_{d}=B
\end{array}
$$

where $B$ is the total budget, $B_{d}$ is the daily budget and $T_{d}$ is the predicted daily average ambience temperature. If the daily budget is not used up, the rest part will be put back to the total budget pool and the HEM will reallocate the budget for future with latest weather prediction. The solution is to fill the daily temperature curve by the budget as in Fig. 8

\section{Slow Time Scale Stochastic Optimization}

We propose a MPC based suboptimal solution to the HEM stochastic problem detail described in Table III, where $T$ is the time interval (one hour in this paper). At the slow time scale, the HEM predicts to the future based on the estimation using Kalman filter with the measurement from the fast time scale and optimizes the hourly power allocation and take only the first step to implement. While solving the stochastic optimization, the noise is replaced by the expectation to reduce the complexity of the computation.

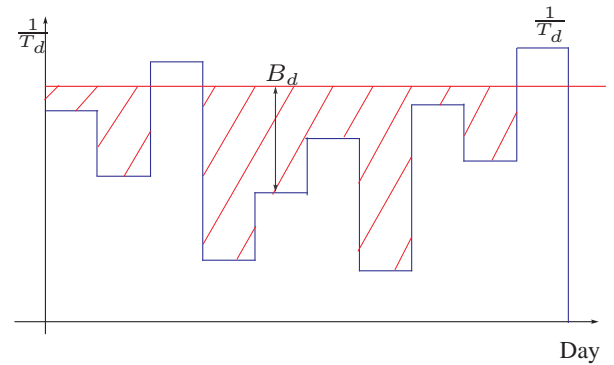

Fig. 8: Water Filling Budget Allocation

TABLE III: Model Predictive Control in Slow and Fast Time Scales

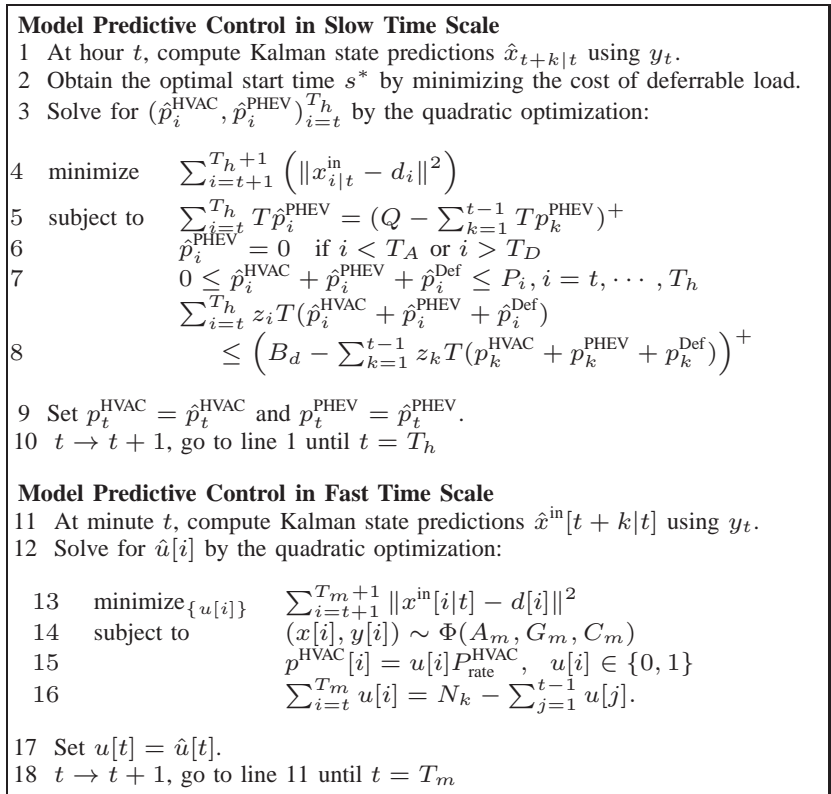

For simplicity, we will assume in the simulation that the deferrable and non-interruptible load draws constant rated power $P_{\text {rate }}^{\text {Def }}$.

\section{E. Fast Time Scale Stochastic Optimization}

The similar MPC principle applies to the fast time scale problem as well, except for some loads we are dealing with integer decision variables.

Considering the control problem for various loads is decomposed by the slow time scale problem, we focus on the control of HVAC at the fast time scale. The most widely used control strategy of the HVAC is the on-off rule based control (RBC), where the heating and the air conditioning is turned on or off by the thermostat according to the actual temperature measurement and the set point [19]. In practice, the length of the interval between HVAC on-off states switching may be an additional constraint as it influences the longevity.

Within the class of integer optimization, we formulate the problem as choosing the on periods to minimize the discomfort level meeting the slow time scale power allocation. Once the switching time is determined, the HEM can implement the 
control of HVAC by artificially changing the set point without actually installing a different controller.

Table III shows the outline of a model predictive control strategy of fast time scale control of HVAC. Assuming that the HVAC system works on the same power level during the working periods, the number of on periods within each hour can be calculated by $N_{k}=\left[p_{k}^{\mathrm{HVAC}} / P_{\text {rate }}^{\mathrm{HVC}}\right]$ with given the total power budget $p_{k}^{\mathrm{HVAC}}$ in the $k$ th hour. The allocation of the on periods is formulated as a stochastic programming with a binary action space. Since different kinds of loads are decomposed in slow time scale problem, other loads can be managed in the similar way. In this paper, the electricity price is assumed constant over one hour, so we omit the fast time scale problem for dryer and PHEV.

\section{Simulation AND PERFORMANCE RESUltS}

Three types of loads were considered in the simulation. As a deferrable and non-interruptible load, the dryer was assumed to start no earlier than 4PM and no later than 8PM, whose working time will last two hours. A PHEV was assumed to be at home from $8 \mathrm{PM}$ to $8 \mathrm{AM}$. The charging rate was set to be nonnegative which implied it could not supply power back to the grid. An hourly peak power constraint, simply assumed to be a sinusoid curve, was applied to the HEM as well as the daily budget constraint.

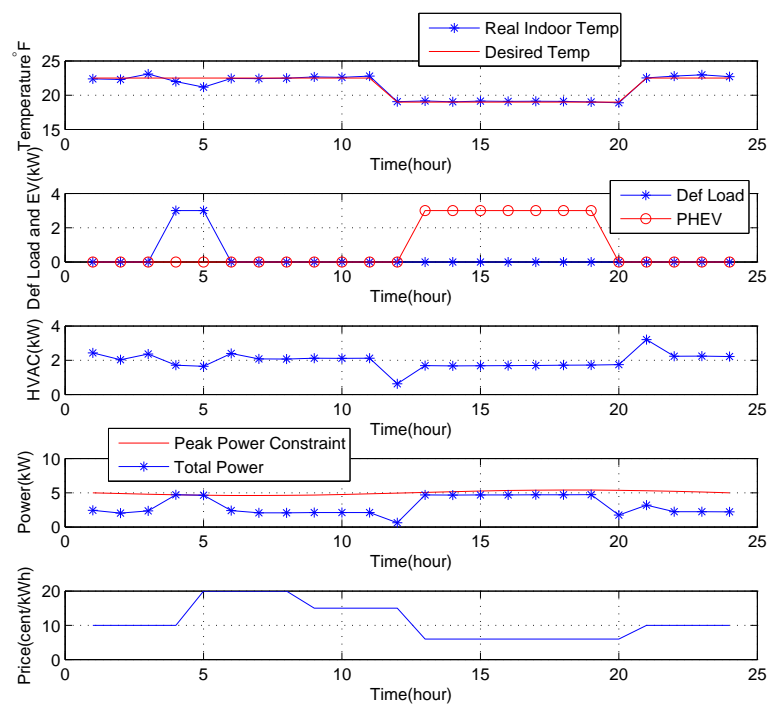

Fig. 9: Power Policy Example: Indoor Temp, Dryer and PHEV Charing, HVAC, Total Power and Peak constraints, Price

The control policy result is illustrated in Fig. 9. Taking the price advantage, the PHEV charging was shifted to the price valley. The dryer, with a tighter schedule deadline, took a relatively low price. Note that the peak power constraint is bounded in some periods. As a consequence, power allocated to HVAC is limited, which caused the indoor temperature deviation from the desired.

To show the impact of MPC approach, we compared the MPC algorithm with a benchmark solution using fabricated thermal parameters and desired temperature. Since the three kinds of loads are combined only in the peak power constraints and the daily budget, we just compare the control of the HVAC, removing other loads from these two constraints. In the simulation, we present the power strategy of the proposed approach and compare it with the performance bound (PB) and the rule based control strategy (RBC) used in the HVAC nowadays. In Fig. 10 we draw the discomfort level comparing with an strategy mixing the MPC and the LQG (thus referred to as MPC-LQG) originally proposed in [11], whose performance will be close to open loop when the constraints are tight and be close to LQG when the constraints are not bounded. The performance bound is obtained by assuming knowing the future weather and system noise perfectly.

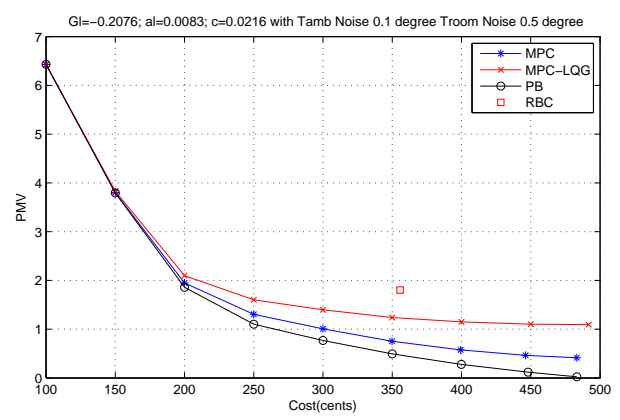

Fig. 10: 1 day performance comparison between Multi-scale Algorithm (MPC), MPC-LQG, PB and RBC

A theoretical simulation was carried out where the outdoor temperature was assumed to follow a auto-regression model. The plot of discomfort level (a measure based on the Predicted Mean Vote (PMV) [20]) against energy cost is shown in Fig. 10. At the PMV level of 1.5, the saving of the proposed approach comparing with MPC-LQG was about $20 \%$. While the MPC spent $8 \%$ more than the performance bound. At the same discomfort level, the multi-scale algorithm spent $30 \%$ less than RBC. Note that when the budget constraint was tight, the difference among the three approaches was small, which can be explained by the fact that all strategies have limited power available for scheduling.

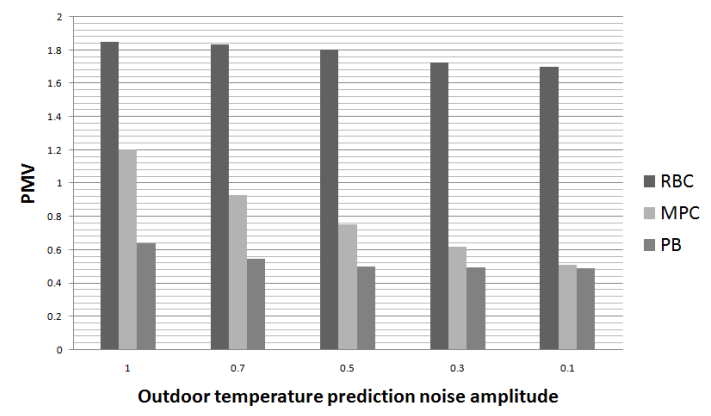

Fig. 11: Performance comparison between RBC, PB and Multi-scale Algorithm (MPC) with various ambient temperature noises

The comparison with a variety of ambience temperature 
prediction noises is shown in Fig. 11. With the same budget, the discomfort level with various noise amplitude are drawn. The simulation result shows that the accuracy of the weather report matters significantly. The gap between MPC and PB increased sharply as the prediction being more noisy.

To make the simulation more close to the realistic, comparison between the proposed multi-scale algorithm (MPC) and the real HVAC performance was carried out based on the real measurement data. In the simulation, the daily dynamic thermal parameters were from the model fitting. With thermal parameters fitted from data of previous day, the HEM allocated the power for the next day, while the indoor temperature changes according to the dynamic equation with the real parameters of the next day. The performance bound was attained by assuming knowing the future weather and system noise perfectly, as well as the dynamic thermal parameters. To show the impact of accuracy of the parameter fitting, the MPC knowing future parameters is plotted as a comparison. The real power consumption of the HVAC using in the house is plotted as one single point in Fig. 12. Because of the lack of set points to the HVAC in the measurements, hourly indoor temperature average is used as the desired temperature. At the same discomfort level, the multi-scale algorithm saves about $12 \%$ comparing to the current HVAC strategy.

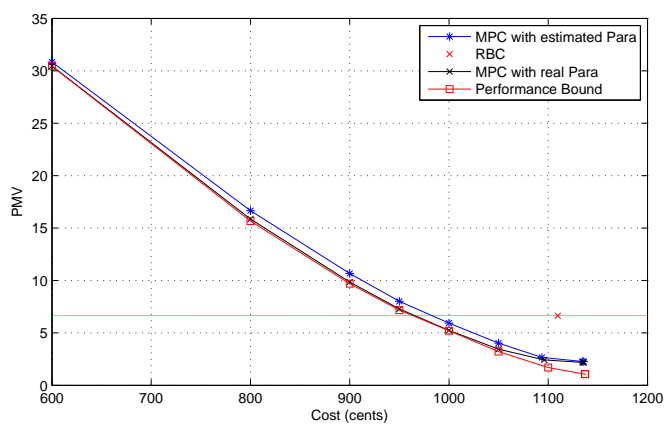

Fig. 12: 10 days performance comparison between RBC , PB and Multi-scale Algorithm (MPC)

\section{CONCLUSION}

We formulate in this paper a multi stage stochastic optimization framework for home energy management system which deals with various characteristic loads. As an interface with the aggregator through real-time pricing signal and economically incentivized power consumption profile, the HEM controls power allocation to minimize the user discomfort level. We propose a multi-scale computationally tractable suboptimal approach to the stochastic programming problem to decompose the control of different types of loads. The linear thermal dynamic model is validated and the online model parameter estimation algorithm is proposed. The comparison with real HVAC performance is carried out.

We have made a number of simplifying assumptions that need to be justified and studied further for practical implementations. More detailed outdoor temperature model should be considered in simulation. The impact of the economically based scheduling on the life-time of appliances, especially in the context of PHEV charging and bang-bang HVAC control, warrants further study. Carefully analysis is needed for budget allocation algorithm. Nonetheless, many of the modifications required to circumvent the above simplifying assumptions can be incorporated into the proposed optimization framework.

\section{REFERENCES}

[1] J. Rawlings, "Tutorial overview of model predictive control," IEEE Control Systems Magazine, June 2000.

[2] R. Sonderegger, "Diagnostic tests determining the thermal response of a house," Lawrence Berkeley National Laboratory, pp. 1 - 15, February 1978.

[3] D. Bargiotas and J. Birddwell, "Residential air conditioner dynamic model for direct load control," IEEE Control Transactions, vol. 3, pp. 2119-2125, October 1988.

[4] S. El-Ferik and R. Malhame, "Identification of alternating renewal electric load models from energy measurements," Automatic Control, IEEE Transactions on, vol. 39, pp. 1184 -1196, jun 1994.

[5] M. Frank, "The kolmogorov-smirnov test for goodness of fit," Lawrence Berkeley National Laboratory, vol. 46, pp. 68 - 78, March 1951.

[6] D. Ha, S. Ploix, E. Zamai, and M. Jacomino, "Tabu search for the optimization of household energy consumption," in 2006 IEEE Int. Conf. Inf. Reuse Integr., pp. 86 - 92, 2006.

[7] M. Pedrasa, T. Spooner, and I. MacGill, "Coordinated Scheduling of Residential Distributed Energy Resources to Optimize Smart Home Energy Services," IEEE Trans. on Smart Grid, vol. 1, September 2010.

[8] D. L. Ha, M. H. Le, and S. Ploix, "An approach for home load energy management problem in uncertain context," in Industrial Engineering and Engineering Management, 2008. IEEM 2008. IEEE International Conference on, pp. $336-339$, dec. 2008.

[9] A. Mohsenian-Rad and A. Leon-Garcia, "Optmal Residential Load Control With Price Prediction in Real-Time Electricity Pricing Environments," IEEE Tran. On Smart Grid, vol. 1, pp. 120-133, Sept 2010.

[10] A. Brissette, A. Hoke, D. Maksimovic, and A. Pratt, "A Microgrid Modeling and Simulation Platform for System Evaluation on a Range of Time Scales," in 2011 IEEE Energy Conversion Congress and Exposition, (Phoenix, Arizona), Sept. 2011.

[11] M. Sznaier and M. J. Damborg, "Suboptimal control of linear systems with state and control inequality constraints," in Decision and Control, 1987. 26th IEEE Conference on, vol. 26, pp. 761 -762, dec. 1987.

[12] N. L. Ricker, "Model predictive control with state estimation," Ind. Eng. Chem. Res., vol. 29, no. 3, pp. 374-382, 1990.

[13] G. Gattu and E. Zafiriou, "Nonlinear quadratic dynamic matrix control with state estimation," Industrial and Engineering Chemistry Research, vol. 31, no. 4, pp. 1096-1104, 1992.

[14] L. Jia, Z. Yu, M. Murphy-Hoye, A. Pratt, E. Piccioli, and L. Tong, "Multi-scale stochastic optimization for Home Energy Management," in Computational Advances in Multi-Sensor Adaptive Processing (CAMSAP), 2011 4th IEEE International Workshop, pp. 113 - 116, Dec. 2011.

[15] A. Hoke, A. Brissette, K. Smith, A. Pratt, and D. Maksimovic, "Electric vehicle charge optimization including effects of lithium-ion battery degradation," in 2011 IEEE Vehicle Power and Propultion Conference, pp. 6-9, Sept. 2011

[16] C. Y. Chong and A. S. Debs, "Statistical synthesis of power system functional load models," in Decision and Control including the Symposium on Adaptive Processes, 1979 18th IEEE Conference on, vol. 18, pp. $264-269$, dec. 1979.

[17] L. McLaughlin, L. Jia, Z. Yu, and L. Tong, "Thermal Dynamic for Home Energy Management: A case study," Tech. Rep. ACSP-TR-10-11-01, Cornell University, Oct. 2011.

[18] J. R. Birge and F. Louveaux, Introduction to Stochastic Programming. 175 Fifth Avenue, New York, NY 10010: Springer-Verlag New York, Inc., 1997.

[19] B. Li and A. Alleyne, "Optimal on-off control of an air conditioning and refrigeration system," in American Control Conference (ACC), 2010, pp. $5892-5897$, July 2010.

[20] D. Ha, S. Zamai, and M. Jacomino, "A home automation system to improve household energy control information control problems in manufacturing," in 12th IFAC Symposium on Information Control Problems in Manufacturing, vol. 12, 2006. 